\title{
A national perspective on prenatal testing for mitochondrial disease
}

\author{
Victoria Nesbitt ${ }^{1}$, Charlotte L Alston ${ }^{2}$, Emma L Blakely ${ }^{2}$, Carl Fratter ${ }^{2,3}$, Catherine L Feeney ${ }^{2}$, \\ Joanna Poulton $^{2,4}$, Garry K Brown ${ }^{2,5}$, Doug M Turnbull ${ }^{1,2}$, Robert W Taylor ${ }^{1,2}$ and Robert McFarland ${ }^{\star, 1,2}$
}

Mitochondrial diseases affect $>1$ in 7500 live births and may be due to mutations in either mitochondrial DNA (mtDNA) or nuclear DNA (nDNA). Genetic counselling for families with mitochondrial diseases, especially those due to mtDNA mutations, provides unique and difficult challenges particularly in relation to disease transmission and prevention. We have experienced an increasing demand for prenatal diagnostic testing from families affected by mitochondrial disease since we first offered this service in 2007 . We review the diagnostic records of the 62 prenatal samples (17 mtDNA and 45 nDNA) analysed since 2007 , the reasons for testing, mutation investigated and the clinical outcome. Our findings indicate that prenatal testing for mitochondrial disease is reliable and informative for the nuclear and selected mtDNA mutations we have tested. Where available, the results of mtDNA heteroplasmy analyses from other family members are helpful in interpreting the prenatal mtDNA test result. This is particularly important when the mutation is rare or the mtDNA heteroplasmy is observed at intermediate levels. At least 11 cases of mitochondrial disease were prevented following prenatal testing, 3 of which were mtDNA disease. On the basis of our results, we believe that prenatal testing for mitochondrial disease is an important option for couples where appropriate genetic analyses and pre/post-test counselling can be provided.

European Journal of Human Genetics (2014) 22, 1255-1259; doi:10.1038/ejhg.2014.35; published online 19 March 2014

\section{BACKGROUND}

Mitochondrial diseases are clinically and genetically heterogeneous conditions affecting $>1$ in 7500 live births ${ }^{1}$ and causing significant morbidity and mortality. ${ }^{2,3}$ Genetic counselling for families with mitochondrial disease can provide some unique and difficult challenges, particularly in relation to disease transmission and prevention. A clear understanding of the inheritance patterns in families with mitochondrial disease, the reproductive and prenatal testing options available, their application to mitochondrial disease, and the risks involved is crucial if accurate and appropriate advice is to be imparted to prospective parents.

Mitochondria are small multifunctional intracellular organelles present in all eukaryotic cells and primarily responsible for generating adenosine triphosphate by oxidative phosphorylation. Their efficient functioning is determined by two genomes; the nuclear genome and also the maternally inherited $16.6-\mathrm{kb}$ mitochondrial genome., ${ }^{4,5}$ Disease may occur as a result of mutations in either of these genomes. Defects in nuclear DNA (nDNA) can cause problems with mitochondrial DNA (mtDNA) maintenance and repair, defects in translation or structural defects of respiratory chain complexes. ${ }^{5,6}$ mtDNA is present in multiple copies within cells and in oocytes the copy number can exceed $1 \times 10^{5}$ per cell. ${ }^{7}$ A consequence of this high intracellular copy number is the phenomenon of heteroplasmy, where two or more species of mtDNA co-exist. This situation arises when a mtDNA mutation occurs within only a proportion of the mtDNA present. Importantly though, disease will only occur when a tissuespecific threshold has been exceeded (usually $>60 \%$ ). This threshold is not the same for all mtDNA mutations and the level of heteroplasmy may change slowly with time., ${ }^{4,5,8,9}$

Patients may develop their first symptoms in adulthood and it is not unusual for the mtDNA mutation to be transmitted to children before their mother becomes symptomatic. In addition, asymptomatic mothers with low levels of mtDNA mutation may have symptomatic children with very high levels because of a genetic phenomenon known as the 'mtDNA genetic bottleneck'. A main component of this bottleneck occurs during formation and initial divisions of primordial germ cells when mtDNA copy numbers are reduced to $<200$ per cell. This decrease in copy number, together with the random segregation of mtDNA molecules to daughter cells, can result in a markedly skewed distribution of mutation when mature oocytes are formed. ${ }^{8,10,11}$

Approximately 30000 women per year are offered invasive prenatal testing in the United Kingdom. ${ }^{12}$ Included in this number is a small, but growing, group of women who are offered prenatal testing for mitochondrial disease. There is no cure for mitochondrial disease and effective treatments are lacking. Reproductive options for families affected by mitochondrial disease are important, particularly those who have already lost a child to the disease or for those women known to harbour mtDNA mutations. Prenatal testing for mitochondrial disease is only possible, however, with known causative mutations, where there is sufficient evidence from segregation and disease linkage studies, biochemical and functional assays to confirm the pathogenicity of these mutations. The primary aim of prenatal diagnosis for mitochondrial disease is to provide an

${ }^{1}$ Wellcome Trust Centre for Mitochondrial Research, The Medical School, Institute for Ageing and Health, Newcastle University, Newcastle-upon-Tyne, UK; ${ }^{2}$ NHS Specialised Services for Rare Mitochondrial Disorders of Adults and Children UK, Oxford, UK: ${ }^{3}$ Oxford Medical Genetics Laboratories, Oxford University Hospitals NHS Trust, Oxford, UK; ${ }^{4}$ Nuffield Department of Obstetrics and Gynaecology, University of Oxford, John Radcliffe Hospital, Oxford, UK; ${ }^{5}$ Department of Biochemistry, University of Oxford, Oxford, UK *Correspondence: Dr R McFarland, Wellcome Trust Centre for Mitochondrial Research, The Medical School, Institute for Ageing and Health, Newcastle University, Framlington Place, Newcastle-upon-Tyne, NE2 4HH, UK. Tel: +44 191282 0340; Fax: +44 191282 4373; E-mail: robert.mcfarland@ncl.ac.uk

Received 6 June 2013; revised 17 December 2013; accepted 16 January 2014; published online 19 March 2014 
accurate assessment of the risk of the foetus developing mitochondrial disease either in utero or in childhood. ${ }^{13-16}$

Where mitochondrial disease is inherited in an autosomal recessive manner, as is most often the case in childhood-onset mitochondrial disease, and the genetic changes identified are novel, then carrier status should be confirmed in each parent with additional evidence provided from functional studies supporting pathogenicity. When a mtDNA mutation is responsible, then heteroplasmy levels in blood and urine should be determined in the mother and, where possible, in maternal relatives, especially previously affected children. For a minority of mtDNA mutations, there is a clear correlation between the level of heteroplasmy and disease severity, but this does differ between families and assessment of foetal risk should be made in the context of how other family members have been affected. ${ }^{16}$

Since establishing the nationally commissioned mitochondrial diagnostic service in 2007, we have experienced an increasing demand for prenatal diagnostic testing from families affected by mitochondrial disease. We have retrospectively reviewed the analyses performed over the period April 2007 to January 2013 to inform the debate and plan future testing strategies.

\section{MATERIALS AND METHODS}

Data regarding prenatal diagnostic testing were collected from two (Newcastle and Oxford) of the three centres (Newcastle, Oxford and London) commissioned by the National Health Service to provide a clinical and diagnostic service for Rare Mitochondrial Diseases of Adults and Children (the service in London did not undertake any prenatal investigations during this period). We are not aware of any other genetic testing centres in the United Kingdom undertaking mtDNA heteroplasmy assessment and although it is possible that other centres have performed prenatal assessment for nuclear mutations, the availability of a free nationally commissioned service makes this somewhat unlikely. Three reviewers examined the diagnostic reports and medical notes over the 69-month period between April 2007 and January 2013. Discrepancies and omissions in the clinical information were clarified with the clinical team.

\section{Assessing mutation status during diagnostic chorionic villus biopsy (CVB) testing \\ A variety of molecular techniques are used for prenatal testing depending upon the causative familial mutation. Presence or absence of nuclear gene mutations is ascertained by direct Sanger sequencing of PCR-amplified products. Prenatal assessment of heteroplasmy levels of familial mtDNA point mutations was achieved using mutation-specific pyrosequencing assays. ${ }^{17}$ These assays have been established in the Newcastle laboratory for a variety of pathogenic mutations and have been shown to be sensitive in their detection of $1 \%$ mutation load. ${ }^{18-21}$ Mutation screening for large-scale single mtDNA deletions is performed using a combined methodology involving quantitative fluorescent real-time PCR (QPCR) and long-range PCR. ${ }^{22}$ The QPCR assay compares the copy number of the commonly deleted MTND4 gene against the copy number of the MTND1 gene, which is seldom deleted in patients with large-scale mtDNA rearrangements. ${ }^{23}$}

\section{RESULTS}

Sixty-two prenatal diagnoses were made in the 69-month study period, of which 59 were CVB samples and 3 were amniocentesis. Gestation at time of testing ranged from 8 weeks 5 days to 15 weeks for CVB, with the amniocenteses being performed between 15 weeks 5 days and 17 weeks 3 days. Prenatal testing was requested in the majority of patients as a consequence of having had a previously affected child $(n=58)$. Other requests were due to being a known mtDNA mutation carrier (although clinically asymptomatic; $n=3$ ), or having severely affected siblings $(n=1)$.

\section{mtDNA mutations}

Of the prenatal samples assessed, 17 were for mutations in $\mathrm{mtDNA} \quad(\mathrm{m} .3243 \mathrm{~A}>\mathrm{G} \quad(n=4), \quad \mathrm{m} .11777 \mathrm{C}>\mathrm{A} \quad(n=2)$, $\mathrm{m} .9176 \mathrm{~T}>\mathrm{C} \quad(n=2), \quad \mathrm{m} .14453 \mathrm{G}>\mathrm{A} \quad(n=1), \quad \mathrm{m} .13513 \mathrm{G}>\mathrm{A}$ $(n=1), \quad \mathrm{m} .8344 \mathrm{~A}>\mathrm{G}(n=1), \mathrm{m} .8993 \mathrm{~T}>\mathrm{G} \quad(n=1), \quad \mathrm{m} .8993 \mathrm{~T}>\mathrm{C}$ $(n=1), \mathrm{m} .10191 \mathrm{~T}>\mathrm{C}(n=1), \mathrm{m} .10158 \mathrm{~T}>\mathrm{C}(n=1), \mathrm{m} .3688 \mathrm{G}>\mathrm{A}$ $(n=1)$ and a single, large-scale mtDNA deletion $(n=1)$; Table 1$)$. In addition to accurately determining the level of mtDNA mutation in the foetal DNA sample, all mtDNA mutations were further categorised as having low $(<30 \%)$, intermediate $(30-70 \%)$ or high $(>70 \%)$ mtDNA loads. Of the 17 prenatal samples, high heteroplasmy levels were detected in CVB samples from patients 6 and 8 and in both cases termination of pregnancy was performed. Patient 8 herself harboured relatively high levels of the m.8993T $>$ G mutation in blood DNA (73\% heteroplasmy) although the threshold for a severe neurological phenotype, such as Leigh Syndrome, in association with the m.8993T $>$ G mutation is typically very high. ${ }^{24}$ In contrast, patient 6 did not have the mutation detectable in her blood or urine. Intermediate heteroplasmy levels were identified in six samples (patients 1, 5, $9,12,14$ and 16), one of which resulted in termination of pregnancy (patient 1), and the outcome for the remainder is not known. Five of these mothers themselves had intermediate heteroplasmy levels of the respective mutation in DNA derived from their urinary epithelium; one had high heteroplasmy levels (patient 9), but (as is the case for the $\mathrm{m} .8993 \mathrm{~T}>\mathrm{G}$ and indeed the m.9176 T $>$ C mutation) the m.8993T $>C$ mutation is associated with a very high threshold. ${ }^{24-26}$ Low level mutation load was detected in the CVB samples from patients 7 and 15, both of whom continued their pregnancies. Patient 15, who harboured low heteroplasmy levels in blood and intermediate levels in buccal and urinary epithelium of m.3688G $>$ A, went on to deliver a clinically unaffected child. The outcome for the child born to patient 7 , in whom we could not detect the familial m.9176T $>C$ mutation in blood or urine, is not known. The familial mutation was not detected in seven prenatal samples, from four mothers in whom we found no evidence of the familial mutation in blood or urine (patient $2(\mathrm{~m} .3243 \mathrm{~A}>\mathrm{G})$, patient $4(\mathrm{~m} .3243 \mathrm{~A}>\mathrm{G})$, patient $10(\mathrm{~m} .14453 \mathrm{G}>\mathrm{A})$, patient 17 (single deletion)), two mothers harbouring low heteroplasmy levels (patient $3(\mathrm{~m} .3243 \mathrm{~A}>\mathrm{G})$ and patient $13(\mathrm{~m} .10191 \mathrm{~T}>\mathrm{C}))$ and one mother with intermediate levels in blood (patient 11 (m.13513G $>$ A)). Postnatal screening for the familial mutation was performed for three cases using blood samples; the results identified no evidence of the corresponding mutation in each instance and they remain clinically well (patient $4(\mathrm{~m} .3243 \mathrm{~A}>\mathrm{G})$, patient 10 (m.14453G $>\mathrm{A})$ and patient $11(\mathrm{~m} .13513 \mathrm{G}>\mathrm{A})$ ).

\section{nDNA mutations}

The remaining 45 prenatal samples were screened for recessive nDNA mutations (POLG $(n=19)$, SURF1 $(n=14)$, NDUFS2 $(n=2)$, MPV17 $(n=2)$, RARS2 $(n=2)$, DGUOK $(n=2), \operatorname{NDUFV1}(n=1)$, TK2 $(n=1)$, SUCLA2 $(n=1)$, TMEM70 $(n=1))$ (Supplementary Table 2). Of these, 12 inherited both parental mutations, 8 of which resulted in termination of the pregnancy, 2 pregnancies continued resulting in the birth of affected infants (mutations confirmed on infant blood DNA for one case) and the outcome of the remaining 2 cases is not known. There were 15 heterozygous carriers and the familial mutations were not detected in 18 cases. Pregnancy was terminated in one of these cases for an incidental finding of the XXY karyotype (patient 26). 
Table 1 Prenatal testing for mtDNA mutations

\begin{tabular}{|c|c|c|c|c|c|}
\hline Pt & Reason for $C V B$ & Screening mutation & Maternal mutation and heteroplasmy level & Result of CVB & Clinical outcome \\
\hline 1 & Mother known mutation carrier & $\mathrm{m} .3243 \mathrm{~A}>\mathrm{G}$ & m.3243A > G (55\% urine, $12 \%$ blood) & $68 \% \mathrm{~m} .3243 \mathrm{~A}>\mathrm{G}^{\mathrm{a}}$ & Termination of pregnancy \\
\hline 2 & Previously affected child & $\mathrm{m} .3243 \mathrm{~A}>\mathrm{G}$ & $\begin{array}{l}\text { m.3243A }>\text { G not detected in blood/urine/ } \\
\text { buccal samples }\end{array}$ & No mutation detected & Pregnancy continued \\
\hline 3 & Mother known mutation carrier & $\mathrm{m} .3243 \mathrm{~A}>\mathrm{G}$ & m.3243A > G ( $1 \%$ blood, $18 \%$ urine) & No mutation detected & Pregnancy continued \\
\hline 4 & $\begin{array}{l}\text { Maternal grandmother and } \\
\text { brother affected }\end{array}$ & $\mathrm{m} .3243 \mathrm{~A}>\mathrm{G}$ & m.3243A > G not detected in blood/urine & No mutation detected & $\begin{array}{l}\text { Baby clinically well; no mutation detected } \\
\text { in blood DNA at } 11 \text { weeks }\end{array}$ \\
\hline 5 & Previously affected child & $\mathrm{m} .8344 \mathrm{~A}>\mathrm{G}$ & m.8344A $>$ G (35\% blood, 35\% urine) & $46 \% \mathrm{~m} .8344 \mathrm{~A}>\mathrm{G}^{\mathrm{a}}$ & Data not available \\
\hline 6 & Previously affected child & $\mathrm{m} .9176 \mathrm{~T}>\mathrm{C}$ & $\mathrm{m} .9176 \mathrm{~T}>\mathrm{C}$ not detected in blood/urine & $98 \%$ m.9176T $>\mathrm{C}^{\mathrm{a}}$ & Termination of pregnancy \\
\hline 7 & Previously affected child & $\mathrm{m} .9176 \mathrm{~T}>\mathrm{C}$ & $\mathrm{m} .9176 \mathrm{~T}>\mathrm{C}$ not detected in blood/urine & $9 \% \mathrm{~m} .9176 \mathrm{~T}>\mathrm{C}^{\mathrm{a}}$ & Pregnancy continued \\
\hline 8 & Previously affected child & $\mathrm{m} .8993 \mathrm{~T}>\mathrm{G}$ & m.8993T>G (73\% blood $)$ & $98 \% \mathrm{~m} .8993 \mathrm{~T}>\mathrm{G}^{\mathrm{a}}$ & $\begin{array}{l}\text { Termination of pregnancy; PM samples } \\
\text { confirmed mutation levels } 97 \%\end{array}$ \\
\hline 9 & Previously affected child & m.8993T $>C$ & $\begin{array}{l}\text { m. } 8993 T>C \text { ( } 79 \% \text { blood, } 86 \% \text { urine, } \\
82 \% \text { buccal) }\end{array}$ & $58 \%$ m.8993T $>C^{a}$ & Pregnancy continued \\
\hline 10 & Previously affected child & m.14453G $>A$ & m.14453G $>$ A not detected & No mutation detected & Clinically unaffected baby girl born \\
\hline 11 & Previously affected child & m.13513A $>$ G & m.13513G >A (45\% blood) & No mutation detected & $\begin{array}{l}\text { Baby clinically well; no mutation detected } \\
\text { in blood DNA at } 12 \text { weeks }\end{array}$ \\
\hline 12 & Maternal brother affected & $\mathrm{m} .11777 \mathrm{C}>\mathrm{A}$ & $\begin{array}{l}\text { m. } 11777 \mathrm{C}>\mathrm{A}(30 \% \text { blood, } 36 \% \text { urine, } \\
43 \% \text { buccal) }\end{array}$ & $46 \%$ m.11777C $>A^{a}$ & Data not available \\
\hline 13 & Mother known mutation carrier & m.10191T >C & m.10191T>C (2\% blood, 16\% urine) & No mutation detected & Pregnancy continued \\
\hline 14 & Previously affected child & $\mathrm{m} .10158 \mathrm{~T}>\mathrm{C}$ & $\begin{array}{l}\text { m. } 10158 \mathrm{~T}>\mathrm{C}(5 \% \text { blood, } 33 \% \text { urine, } \\
16 \% \text { buccal) }\end{array}$ & $52 \%$ m.10158T > C & Data not available \\
\hline 15 & Previously affected child & m.3688G $>A$ & $\begin{array}{l}\text { m.3688G }>\text { A ( } 20 \% \text { blood, } 50 \% \text { urine, } \\
43 \% \text { buccal) }\end{array}$ & $3 \% m .3688 G>A^{a}$ & Clinically unaffected baby girl born \\
\hline 16 & Previously affected child & $\mathrm{m} .11777 \mathrm{C}>\mathrm{A}$ & $\begin{array}{l}\text { m. } 11777 \mathrm{C}>\mathrm{A} \text { ( } 18 \% \text { blood, } 32 \% \text { urine, } \\
20 \% \text { buccal) }\end{array}$ & $54 \% \operatorname{m} .11777 \mathrm{C}>\mathrm{A}^{\mathrm{a}}$ & Alive and well at 5 years \\
\hline 17 & Previously affected child & $\begin{array}{l}\text { Single large-scale } \\
\text { mtDNA deletion }\end{array}$ & No deletion in mtDNA detected & $\begin{array}{l}\text { mtDNA deletion not } \\
\text { detected }\end{array}$ & Pregnancy continued \\
\hline
\end{tabular}

Seventeen women referred for prenatal testing because of a personal or maternal family history of mtDNA disease, results of the prenatal test and clinical outcome where known.

Reference sequence: Revised Cambridge Reference Sequence; 35 GenBank Reference NC_012920.1.

anformation submitted to the publicly available MITOMAP database (http://www.mitomap.org/MITOMAP).

\section{DISCUSSION}

The aim of prenatal diagnostic testing in mitochondrial disease is to identify foetuses harbouring mutations that will cause severe disease and offer termination at a relatively early stage of pregnancy. In our experience, prenatal testing is usually requested as a consequence of having had a previously affected child (57/62 requests).

CVB, usually performed at 11-14 weeks gestation, is the procedure most commonly offered to women at risk of having a child affected by mitochondrial disease, and particularly for those harbouring mtDNA mutations. Amniocentesis, a procedure that relies on aspirating cellular material (largely shed foetal epithelial cells) from the amniotic sac, may be less reliable in detecting and quantifying mtDNA mutations and tends to be offered to those women presenting later in pregnancy or where a nDNA mutation is responsible for the mitochondrial disease. Prenatal diagnostic testing procedures also bring additional risk of miscarriage; approximately $1 \%$ and $2 \%$ higher than spontaneous miscarriage for amniocentesis and CVB, respectively. ${ }^{12}$ No spontaneous miscarriages occurred as a result of prenatal testing in our cohort. Percutaneous umbilical blood sampling is not routinely offered in mitochondrial disease because of the risk of maternal contamination of the sample and the increased risk of foetal loss $(<3 \%)$ associated with the procedure. ${ }^{12}$ We do not offer a biochemical prenatal diagnosis, although this has been performed (rarely) at other international centres where a biochemical deficiency has been established in a previously affected child, but no genetic diagnosis achieved. This type of biochemical diagnosis has a number of specific requirements and as a result worldwide experience is very limited. ${ }^{16}$
For women known to harbour pathogenic mtDNA mutations, interpretation of prenatal testing is complex and it is important to consider factors such as heteroplasmy of the mtDNA mutation, threshold levels, phenotypic expression of the mutation in maternal relatives and the strength of association between genotype and phenotype. Only through evaluating all of these aspects can the clinician provide clear, accurate genetic advice regarding the likely outcome for the foetus affected by a mtDNA mutation. ${ }^{13,15,16,20}$ Recent studies indicate that prenatal samples provide an accurate prediction of mutation load in the postnatal period. ${ }^{27}$ In addition, studies analysing heteroplasmy levels in blastomeres from disaggregated eight cell stage embryos, have indicated that there is less variation between cells than was previously considered to be the case, implying that sampling bias in prenatal diagnosis is minimal. ${ }^{28}$ Further research to ascertain the correlation between genotype, mutation load and phenotype is required with regards to some mtDNA mutations. In the seven cases in which the mutation was not detected, these families could be reassured that their child would not be clinically affected by mitochondrial disease. Counselling mothers with foetuses harbouring mtDNA mutations in the intermediate range $(n=11)$ can prove more difficult particularly for mutations where the relationship between genotype and phenotype is far less clear (eg, m.3243A > G). In this context, information regarding levels of heteroplasmy in affected and unaffected close relatives is very important. This was particularly true for patient 1 (Table 1), who was the only intermediate result to lead to termination of pregnancy. Extensive pre- and post-result counselling included a discussion of $\mathrm{m} .3243 \mathrm{~A}>\mathrm{G}$ heteroplasmy levels present in patient 1's affected and 
unaffected maternal relatives, who gave permission to disclose this information for the purposes of counselling. The two women with foetuses demonstrating high heteroplasmy levels on prenatal testing both opted for termination of pregnancy following appropriate counselling with both a specialist in mitochondrial disease and a genetic counsellor. Although all families affected by mitochondrial disease should be aware of available reproductive options, it is these women, harbouring high levels of pathogenic mtDNA mutations, who may wish to consider alternative options for future pregnancies. Such options include gamete (egg or sperm) donation, or adoption as an alternative to pregnancy. Techniques to reduce or prevent transmission of mtDNA and nDNA mutations, such as pre-implantation genetic diagnosis, are already available. ${ }^{14,29,30}$

One of the shortfalls of this retrospective collection of data on prenatal diagnoses in patients with mitochondrial disease is the paucity of information available regarding follow-up on children born after prenatal diagnosis. It appears that for mothers with low or intermediate levels of mtDNA mutation, routine outpatient follow-up is infrequent and therefore information regarding their children scant. However, as a national service, we are not aware of any children presenting with mitochondrial disease symptoms following prenatal mtDNA diagnosis during the period April 2007-January 2013.

Interpretation of prenatal testing is relatively straightforward in those families harbouring nDNA mutations where classic Mendelian rules of inheritance apply. Parents can be given much clearer results with regards as to whether or not their child will be affected. In keeping with current UK practice, at parental request we have also provided information on the carrier status of the foetus. ${ }^{31}$

For homoplasmic maternally transmitted mtDNA disease, where PGD is not an option, further strategies are being developed. Techniques such as pronuclear transfer, where male and female pronuclei from the patient's fertilised oocyte are transferred to an enucleated healthy fertilised donor oocyte, and metaphase II spindle transfer, which involves the transfer of the metaphase II spindle from a mature affected oocyte into an enucleated healthy donor oocyte before fertilisation, are at an advanced stage of development with successful metaphase II spindle transfer having been performed in Macaque monkeys. ${ }^{32-34}$ In March 2013, the Human Fertilisation and Embryology Authority (HFEA) agreed its advice to UK Government on the ethics and science of IVF-based techniques designed to prevent the transmission of maternally inherited mitochondrial disease. Further work assessing the safety, efficacy and applicability of this technique is planned (www.hfea.gov.uk/6896.html).

\section{CONCLUSION}

We have experienced an increasing demand for prenatal diagnostic testing from families affected by mitochondrial disease, with 62 prenatal diagnoses made since the service was first offered in 2007 and at least 11 cases of mitochondrial disease prevented.

Information on reproductive options including prenatal diagnosis (CVB or amniocentesis), egg donation and sperm donation, techniques to reduce or prevent transmission (eg, PGD), or adoption as an alternative to pregnancy, should be presented to all families affected by mitochondrial disease to facilitate informed reproductive choices. Pronuclear transfer and metaphase II spindle transfer continue to be developed, but are not yet available. The present availability of PGD and the future possibility of nuclear transfer mark an exciting phase in preventing the transmission of mitochondrial disease.

\section{CONFLICT OF INTEREST}

The authors declare no conflict of interest.

\section{ACKNOWLEDGEMENTS}

$\mathrm{VN}$ is a clinical research associate funded through the Medical research Council Mitochondrial Disease Patient Cohort Study UK (G0800674). RMcF is funded by a HEFCE/Department of Health Clinical Senior Lectureship. RMcF, RWT and DMT are PIs in the MRC Centre for Neuromuscular Diseases (G0601943). DMT and RWT are also funded by a Wellcome Trust Strategic Award (906919). CLA is supported by a NIHR doctoral research fellowship. We are grateful to the patients and their referring clinicians for the data provided in this study. We are also grateful to all staff involved in the prenatal diagnoses carried out at Oxford Medical Genetics laboratories, in particular Dr Conrad Smith and Dr Julie Evans, and to Jo Lowndes, Genetic Counsellor. All mtDNA and nDNA mutations described in the article have been submitted to publicly available databases: MITOMAP (http://www.mitomap.org/MITOMAP), the Human DNA Polymerase Gamma Mutation Database (http://tools.niehs. nih.gov/polg/) and the Leiden Open Variation Database (LOVD) 3.0 Shared Installation (http://www.lovd.nl/GENESYMBOL).

1 Skladal D, Halliday J, Thorburn DR: Minimum birth prevalence of mitochondrial respiratory chain disorders in children. Brain 2003; 126: 1905-1912.

2 Scaglia F, Towbin JA, Craigen WJ et al: Clinical spectrum, morbidity, and mortality in 113 pediatric patients with mitochondrial disease. Pediatrics 2004; 114: 925-931.

3 Schaefer AM, McFarland R, Blakely EL et al: Prevalence of mitochondrial DNA disease in adults. Ann Neurol 2008; 63: 35-39.

4 Taylor RW, Turnbull DM: Mitochondrial DNA mutations in human disease. Nat Rev Genet 2005; 6: 389-402.

5 Schon EA, DiMauro S, Hirano M: Human mitochondrial DNA: roles of inherited and somatic mutations. Nat Rev Genet 2012; 13: 878-890.

6 Battersby BJ, Loredo-Osti JC, Shoubridge EA: Nuclear genetic control of mitochondrial DNA segregation. Nat Genet 2003; 33: 183-186.

7 Poulton J, Marchington DR: Segregation of mitochondrial DNA (mtDNA) in human oocytes and in animal models of mtDNA disease: clinical implications. Reproduction 2002; 123: 751-755

8 Cree LM, Samuels DC, de Sousa Lopes SC et al: A reduction of mitochondrial DNA molecules during embryogenesis explains the rapid segregation of genotypes. Nat Genet 2008; 40: 249-254.

9 Marchington DR, Scott-Brown M, Barlow DH, Poulton J: Mosaicism for mitochondria DNA polymorphic variants in placenta has implications for the feasibility of prenatal diagnosis in mtDNA diseases. Eur J Hum Genet 2006; 14: 816-823.

10 Jacobs L, Gerards M, Chinnery $\mathrm{P}$ et al: mtDNA point mutations are present at various levels of heteroplasmy in human oocytes. Mol Hum Reprod 2007; 13 149-154.

11 Carling PJ, Cree LM, Chinnery PF: The implications of mitochondrial DNA copy number regulation during embryogenesis. Mitochondrion 2011; 11: 686-692.

12 Royal College of Obstetrics and Gynaecologists. Amniocentesis and Chorionic Villus Sampling. Green-top Guideline No. 8 June 2010.

13 Bredenoord A, Dondorp W, Pennings G et al: Preimplantation genetic diagnosis for mitochondrial DNA disorders: ethical guidance for clinical practice. Eur J Hum Genet 2009. 17: 1550-1559.

14 Dean NL, Battersby BJ, Ao A, Gosden RG, LinTan S, Shoubridge EA: Prospect of preimplantation genetic diagnosis for heritable mitochondrial DNA diseases. Mol Hum Reprod 2003; 9: 631-638.

15 Jacobs LJ, de Coo IF, Nijland JG et al: Transmission and prenatal diagnosis of the T9176C mitochondrial DNA mutation. Mol Hum Reprod 2005; 11: 223-228.

16 Thorburn DR, Dahl HH: Mitochondrial disorders: genetics, counseling, prenatal diagnosis and reproductive options. Am J Med Genet 2001; 106: 102-114.

17 Ronaghi M, Uhlen M, Nyren P: A sequencing method based on real-time pyrophosphate. Science 1998; 281: 363-365.

18 Alston CL, Lowe J, Turnbull DM, Maddison P, Taylor RW: A novel mitochondrial tRNAGlu (MTTE) gene mutation causing chronic progressive external ophthalmoplegia at low levels of heteroplasmy in muscle. J Neurol Sci 2010; 298: 140-144.

19 Alston CL, He L, Morris AA et al: Maternally-inherited mitochondrial DNA disease in consanguineous families. Eur J Hum Genet 2011; 19: 1226-1229.

20 Lax NZ, Gnanapavan S, Dowson SJ et al: Early-onset cataracts, spastic paraparesis, and ataxia due to a novel mitochondrial tRNAGlu (MT-TE) gene mutation causing severe complex I deficiency: a clinical, molecular and neuropathological study. J Neuropath Exp Neurol 2013; 72: 164-175.

21 Blakely EL, Yarham JW, Alston CL et al: Pathogenic mitochondrial tRNA point mutations: nine novel mutations affirm their importance as a cause of mitochondrial disease. Hum Mutat 2013; 34: 1260-1268

22 Blakely EL, He L, Taylor RW et al: Mitochondrial DNA deletion in 'identical' twin brothers. J Med Genet 2004; 41: e19.

$23 \mathrm{He}$ L, Chinnery PF, Durham SE et al: Detection and quantification of mitochondria DNA deletions in individual cells by real-time PCR. Nucleic Acids Res 2002; 30: e68.

24 White SL, Collins VR, Wolfe R et al: Genetic counseling and prenatal diagnosis for the mitochondrial DNA mutations at nucleotide 8993. Am J Hum Genet 1999; 65 474-482. 
25 Dionisi-Vici C, Seneca S, Zeviani M et al: Fulminant Leigh syndrome and sudden unexpected death in a family with the T9176C mutation of the mitochondrial ATPase 6 gene. J Inherit Metab Dis 1998; 21: 2-8.

26 Campos Y, Martín MA, Rubio JC et al: Leigh syndrome associated with the T9176C mutation in the ATPase 6 gene of mitochondrial DNA. Neurology 1997; 49: 595-597.

27 Marchington D, Malik S, Banerjee A et al: Information for genetic management of mtDNA disease: sampling pathogenic mtDNA mutants in the human germline and in placenta. J Med Genet 2010; 47: 257-261.

28 Steffann J, Frydman N, Gigarel N et al: Analysis of mtDNA variant segregation during early human embryonic development: a tool for successful NARP preimplantation diagnosis. J Med Genet 2006; 43: 244-247.

29 Brown DT, Herbert M, Lamb VK et al: Transmission of mitochondrial DNA disorders: possibilities for the future. Lancet 2006; 368: 87-89.

30 Poulton J, Turnbull DM: 74th ENMC International Workshop: mitochondrial diseases. Neuromuscul Disord 2000; 10: 460-462.

31 Royal College of Physicians, Royal College of Pathologists and British Society for Human Genetics. Consent and confidentiality in clinical genetic practice: guidance on genetic testing and sharing genetic information. 2nd edn. Report of the Joint Committee on Medical Genetics. London: RCP, RcPath 2011.

32 Craven L, Tuppen HA, Greggains GD et al: Pronuclear transfer in human embryos to prevent transmission of mitochondrial DNA disease. Nature 2010; 465 : 82-85.

33 Tachibana M, Amato P, Sparman M et al: Human embryonic stem cells derived by somatic cell nuclear transfer. Cell 2013; 153: 1-11.

34 Tachibana M, Amato P, Sparman M et al: Towards germline gene therapy of inherited mitochondrial diseases. Nature, 493: 627-631.

35 Andrews RM, Kubacka I, Chinnery PF et al: Reanalysis and revision of the Cambridge reference sequence for human mitochondrial DNA. Nat Genet 1999; 23: 147.

\section{(c) (i) This work is licensed under a Creative Commons Attribution 3.0 Unported License. To view a copy of this license, visit http://creativecommons.org/licenses/by/3.0/}

Supplementary Information accompanies this paper on European Journal of Human Genetics website (http://www.nature.com/ejhg) 